\title{
Confirmatory Research on a Representative Method of Describing Knowledge Task Process
}

\author{
Huan Cao \\ School of Management and Economics, University of Electronic Science and Technology of China \\ Chengdu 610054, China \\ E-mail: cigo_ch@163.com \\ Yongjian Li \\ School of Economics and Management, Southwest Jiaotong University, China \\ Chengdu 610031, China
}

This research paper was supported by the Natural Science Foundation of China Grant 70871099.

\begin{abstract}
This paper presents research findings that knowledge task process representation can be used to enhance the efficiency of knowledge tasking. This representative knowledge tasking can simulate knowledge tasking in the same way used in artificial intelligence and knowledge engineering. It can describe the process of how to solve the Tower of Hanio in predicate logic, and analyze the problems that exist in knowledge representation. Based on cognitive science it can advance the approach to represent knowledge in Mind Operation (MO) by case study and use confirmatory factor analysis to validate the ubiquity of MO. The data supports the 9 element mind operation concept set. It further provides a basic concept and methodology to measure and optimize tasks, and explores a way of knowledge representation in AI.
\end{abstract}

Keywords: Knowledge task, Mind operation, Knowledge task process representation, Knowledge representation, Confirmatory factor analysis

\section{Introduction}

Today knowledge itself creates wealth. Drucker states that increasing and improving the efficiency of knowledge work is "the necessary requirement to survive in developed countries" (Peter F. Drucker, 1999). He also emphasized that "improving the efficiency of knowledge work will be the primary task in the new century ... as the improvement of the efficiency of manual work was in the last century" (Peter F. Drucker, 1999). The research on improving productivity of knowledge workers has just begun. Today, what we know about it is as much as the research on manual worker productivity was one century ago (Peter F. Drucker, 1999). Even the concepts of knowledge work and knowledge workers are still not unanimous (Li Yong-jian, Ma Shun-dao, 2004). The research on this field and especially about how to manage the process of knowledge work has advanced very slowly (Thomas H. Davenport, Robert J. Thomas, Susan Cantrell, 2002).

The brain process has traditionally been treated as a "black box" in management research and practice. Most research focuses on how to support knowledge work, such as promoting, group management, and the environment of knowledge work (Russell Curtis \& David Leon, 2002; Tomas Hellstroèma, Ulf Malmquistb, Jon Mikaelssonc, 2001; E.Kevin Kelloway \& Julian Barling, 2000). The mental labor management based on the forward feedback (the competent traits) and feedback (the promoting and control), lacks the process control and will inevitably result in the phenomena such as "Philippe" software crisis (the efficiency of the work is inverse ratio with the cube root of the size of group) (Li Yong-jian, Ma Shun-dao, 2004). To solve the problem resulting from the lack of process management, we must shift from the goal management to the basic logic followed by scientific management: control the result by controlling the process (Li Yong-jian, Ma Shun-dao, 2004). The control and management of the process is the foundation of scientific 
management. The primary problem we need to address is how to describe and represent the process of knowledge work. This is a fundamental problem related to the brainwork process or "black box" (Wang lei, Li Yong-jian, 2006).

In the middle of the last century, after the behaviorist Edward C. Tolman advanced the term "medium variables". Psychologists then begun to explore the inner process of the mind, trying to uncover the "black box". Cognitive psychology research was conducted on cognition and intelligence. Not only the intuitive and philosophical discussion, but also studies based on modern scientific approaches (Chen Lin, 1982\&1993).

\section{What is knowledge task process?}

The definition we give to knowledge tasking is based on the following conceptions:

1) Accepting the modern scientific definition of knowledge levels and conditions, we can define knowledge tasking as "complex and non-observed intelligence in the human brain" or regard it as "creationary intellective activity," or "insight". We therefore regard knowledge task processing as a "black box".

2) If we regard knowledge task as a wealth creating process, from the point of view of economics, neither research nor representation is necessary for knowledge task processing.

3) If we think human work is all the activities dominated by the brain and regard knowledge tasks as "more mental labor, less manual work", then it would get into the dissension of "more or less" while ignoring the discussion of the essential characteristics of knowledge process.

In order to represent the knowledge task process effectively, the knowledge task definition used in this research is not intuition of creative mental labor but the sequential process of mechanical mental labor. This definition facilitates the study's research and analysis of the process of knowledge tasking.

2.1 From the Cognitive psychology point of view knowledge tasking is the human brain's operating process using subjective symbols

Undoubtedly, knowledge tasking is intellectual activity processing. The description of knowledge tasking equates to the description of human intelligence as activity processing. As long ago as the beginning of $20^{\text {th }}$ century, Thorndike proposed a "special factor theory". It suggests that intelligence is comprised of many special capacities, and he inaugurated research on how to describe and interpret human intellective activities. Human intellective activities are very complex phenomenon that has produced numerous research. This paper hopes to contribute to several of these theories related to knowledge task.

Guilford brought forth his "three-dimension" theory of intelligence in 1959. This theory considered that intelligence was a three-dimensional space structure comprised of operations (thinking methods, including five components: cognition, memory, divergent thinking, converge thinking, evaluating) $\times$ contents (objects which thinking operates on: figure, symbol, semantic meaning, action) $\times$ results (outcomes when certain operation acts on certain content: cell, kind, relation, system, transform, meanings). In this three-dimension structure, intelligence is not only mind operation, but also a three-dimension body that has close relationships with operational objects and operational results.

In the middle of the 1970's, the cognitive revolution began to impact on the research field of intelligence. The most representative research was Sternberg's Triarchic, "Theory of Human Intelligence" and the PASS theory put forward by Das. Their consensus in intelligence research was a transforming focus from traits analysis to interior process. They depicted the mind mechanism of intelligence operation by way of the cognitive process. They used the process analysis method of information-process, and described the interior process of intellective activities more quantitatively.

A component was the basic cell of intelligence operation analysis in Sternberg's Triarchic Theory of Human Intelligence. Sternberg thought was that a "component is a type of basic information processing. It operates on the basis of interior representation of objects or symbols. Such a process can transform a sensation input into a concept representation; it also can transform a concept representation into a movement output" (Sternberg R.J., 1985). Sternberg divided components into metacomponents, performance components and knowledge-acquisition components. Metacomponent was the highest-level control process. It played a role on constituting plans, choosing strategy, supervising execute, appraising results and adjusting feedback. The performance component was the component that a subject used when he implemented task operations with various strategies. Its function was implementing the dictates of metacognition and carrying out various idiographic cognitive process operations. Some performance components would only be used in a multitude of special tasks. Universal performance components, such as coding, combination and comparing of stimulating, inference etc., gained more attention (Sternberg R.J., 1985). Those could be viewed as basic mind operations, or mind operations at the "therblig" level.

Cognitive psychologist therefore regards knowledge tasking as the human brain's mind operating process on subjective symbols. 
2.2 From the cognitive science and artificial intelligence point of view; knowledge tasking is the human brain's information and calculating process

Artificial intelligence compares the brain to the computer. It regards the mind's process as being similar to computer processing; however, it explains the mind's process as more delicate and elaborate (Fu Xiao-lan, Liu Chao, 2003). It uses basic rules in a physical symbol system to explain the mind's complicated psychological phenomena and regards the process of solving problems as information and calculating processing (Simon H.A, 1983).

Nobel Prize winner Simon, renowned for his work in cognitive science, regarded the mind's operation as the calculating process of human brain. The human brain's operation or processing uses certain symbols. This transformation of semantic symbols are restricted by rules. For example, the addition formula, it mainly formed vision-Arabic numerals representation and audition-verbal representation of the multiplication formula (Zhou Xin-lin, Dong Qi, 2003). It could be said that in the sense of symbol transformation, whether human brain or computer, all form a system of symbol operation and processing. The human brain's cognition and intelligence activities can all be simulated through computer coding into symbols (Yan Ping-fan, Zhang Chang-shui, 2002). Therefore, "the human brain is a computer, thinking is calculation, cognition is symbol calculation" (Yan Ping-fan, Zhang Chang-shui, 2002).

In the abstract, computing is the mapping or transformation based on a symbolic string. Computing is the process of changing an existing string into a presupposed string by limited steps. The computing process function simply transforms the strings, e.g., transforming " $1+1$ " into 2 is an addition calculation, and $\mathrm{x}^{2}$ into $2 \mathrm{x}$ is differential coefficient calculation. Theorem proving, letter translation, numerical calculation and non-numerical calculation are all computing. Transforming the poem "flocks and herds appear as grass bends to wind" into an image of grazing livestock is also a kind of computing. It transforms literal information symbols into image information symbols. The algorithm of this computing is called mind operation. Such transformation is fulfilled by the human brain through the mind operation of "reproductive imagination".

Cognition information processing theory has five basic theoretic hypotheses. Recursive decomposition hypothesis states that any complicated (un-original) information event could decompose into lower level and more detailed components to describe it. In other words the cognitive process consists of more simple cognitive processes (these processes also could be decomposed into much more simple cognitive process), and any knowledge task process also could be decomposed into more simple mind operations and described by mind operation chains. Adelaman (American South-California University) first published the DNA computer theory in the journal of "Science" in 1994. The DNA computer theory proposes that cognition and thinking are kinds of recursive computing in molecule level. So, knowledge tasking is similar to information processing. It is symbol transformation by the process of the brain's computing operation.

Obviously, in the point of view of cognitive science and artificial intelligence, knowledge task process is human brain's information processing of subjective symbols within the brain. It is human brain's calculating process.

2.3 From the scientific management point of view, mind operation is the "therblig" of knowledge tasking - a succession of mind operations which compose knowledge task processing

Before the concepts of "basic human actions" defined as "therblig" has brought forward, human manual work was in the state of the "gray box". In 1881, Taylor found that "basic motion" in a certain industry also exists in other industries (Frederick W. Taylor, 1911). At the same time, through research on actions in the brickwork industry, Gilbreth put forward a concept he called "therblig", which is similar with "basic motion". Using the concepts of "basic motion" and "therblig", Taylor defined manual work as "a series of actions and work is only a series of simple actions". Since manual operations tasks are only a series of simple actions, the processes of different types and nature of operations tasks can also be interpreted as "motion" or "therblig". To transform consecutive manual work or operations task processes to discrete process comprised of "motions" or "therbligs" - the method is called "motion and time studies". It makes operations task become a controllable process whose method can be optimized and measured in time. Consequently, the origin of Science Management was established.

Operations tasks are operational processes that human physical forces act on physical objects, likewise, knowledge tasks are operational processes that human mental efforts act on symbol information. So, the processes that human mental efforts act on symbol information, and then at a higher-level consecutive knowledge task process, can be transformed into a discrete process. This is comprised of a series of "basic motions" and interpreted as "therbligs". The motions of operation tasks are muscles and bone activities. The knowledge task's "motions" and "therbligs" could be regarded as the mind operations of the human brain. Similar to defining manual work and operation tasks by "basic motion" and "therblig", we can describe knowledge task process effectively with mind operations.

So, from the point of view of scientific management, knowledge is a series of mind operation processes. 


\subsection{Knowledge tasking is the human brain's mind operating process on subjective symbol}

In summary, from the "process" point of view knowledge tasking is the human brain's succession of mind operating processes on subjective symbol. Such process is mechanical, ordinal, calculable and form-repeated (not semantics and content repeated) process (Li Yong-jian, Ma Shun-dao, 2004).

It needs to be explained that this paper only discusses what knowledge task process is, not why. That is the field of cognitive science. So, the manners of mental labor (thinking, cognition or information processing manners including "inspiration" and "insight"), all could be regarded as discrete points or the "therbligs" of mind operation. This definition avoids the difficulties of description and research caused by the problems such as "inspiration". Because the goal of this paper is to demonstrate the use of limited mind operation concepts to describe limitless different knowledge task processes. This could be used to optimize task methods, measure task time, and subsequently improve management efficiency.

\section{The formalization of the process of knowledge tasking}

The object of our research focuses on the formal representation of knowledge work process. We explore the problem in two aspects: artificial intelligence and psychology.

\subsection{Knowledge representation of knowledge task processing}

Artificial intelligence is based on the simulation of the human brain's working principles. We also can analyze the knowledge working process by analyzing the knowledge representation. The problem solving process advanced by Newell and Simon created the formal research into artificial intelligence in 1950s. Research in artificial intelligence has spread into two fields: Symbolism and Connectionism. Symbolism emphasizes the functional simulation and regards the basic unit of intelligence as symbols. The cognitive process is symbol computing by symbol representation. This is the traditional way to study artificial intelligence as psychological symbol processing. Connectionism emphasizes the structural simulation, using the neural-psychological method that is the method of artificial neural network (Liu Zhong-pei, Sun Jing, 2001). These two ways to study artificial intelligence is the new trend in amalgamation: the manipulation of symbols is realized in the sphere of connectionism (Wei Hui, Pan Yun-he, 2000). The aim of artificial intelligence is to let a machine fulfill the work that needed the intelligence of human beings (Minsky M, 1968). The key to have the machine solve the problem as a human brain is how to include the experts' experience and how to represent it in the acceptable and useable way by computer (Feng Ding, 2006). The knowledge representation is the foundational problem in artificial intelligence. Predicate Logic, procedural Rule, Frame and Semantic Network are the popular knowledge representations in artificial intelligence (Feng Ding, 2006).

If we regard the computer as the physical realization of symbolic system, we could formalize the problem to be solved. Formalization is to represent the objects as uniform abstract symbols and set rules. In a broad sense, formalization refers to all the objects being sensed and combined. They need to find the corresponding material in the consciousness (such as images and sounds). The mental activity is fulfilled by organization of these materials. In narrow sense, the formalization is to set up a series of algorithms that can be carried out by the computer (Wang Qian-ling, 2004).

So the representation of the knowledge task process is the formalization of the process. We use predicate logic to describe the typical program problem "Tower of Hanio" as followed (Tan Hao-qiang, 1999).

\subsubsection{Using predicate logic representation to solve the process of the "Tower of Hanoi"}

The problem is as follows: there was a tower in ancient time, and 3 sets of plates are located in the tower. To begin with the set b1 had 64 plates. The plates were different, and the bigger ones are always under the smaller ones. (See the fig.1) An old monk wanted to transfer the 64 plates from the set b1 to set b3. He is only permitted to transfer one plate at a time. During the transfer process the bigger ones should always be under the small ones in the 3 sets.

Insert Figure 1 here

Suppose the $a_{i}$ as plates, $i=1,2,3, \ldots n, \quad a_{i+1}>a_{i}$; For explaining it more clearly, we take only 3 plates $(n=3)$

Suppose the $b_{1}, b_{2}, b_{3}$ as the 3 poles.

Suppose the $\mathrm{U}$ as "on...pole, and...is above..." $\mathrm{U}\left(\mathrm{b}_{1}, \mathrm{a}_{1}, \mathrm{a}_{2}\right)$ indicates is on the b1pole and alis above a2.

Suppose the $\mathrm{M}$ as “...was transferred from...to..." $\mathrm{M}(\mathrm{a} 1, \mathrm{~b} 1, \mathrm{~b} 2)$ indicates that $\mathrm{a}_{1}$ was transferred from $\mathrm{b}_{1}$ to $\mathrm{b}_{2}$. $\mathrm{U}$ and $\mathrm{M}$ can describe the whole process of transfer.

(0) initial: U (b1, a1, a2), U (b1, a2, a3), U (b2, 0, 0), U (b3, 0, 0);

(1) step: $\mathrm{M}(\mathrm{a} 1, \mathrm{~b} 1, \mathrm{~b} 3)$, and $\mathrm{U}(\mathrm{b} 1, \mathrm{a} 2, \mathrm{a} 3), \mathrm{U}(\mathrm{b} 2,0,0)$; $\mathrm{U}(\mathrm{b} 3, \mathrm{a} 1,0)$;

(2) step: $\mathrm{M}(\mathrm{a} 2, \mathrm{~b} 1, \mathrm{~b} 2)$, and $\mathrm{U}(\mathrm{b} 1, \mathrm{a} 3,0), \mathrm{U}(\mathrm{b} 2, \mathrm{a} 2,0), \mathrm{U}(\mathrm{b} 3, \mathrm{a} 1,0)$;

(3) step: $\mathrm{M}(\mathrm{a} 1, \mathrm{~b} 3, \mathrm{~b} 2)$ and $\mathrm{U}(\mathrm{b} 1, \mathrm{a} 3,0), \mathrm{U}(\mathrm{b} 2, \mathrm{a} 1, \mathrm{a} 2), \mathrm{U}(\mathrm{b} 3,0,0)$; 
(4) step: $\mathrm{M}(\mathrm{a} 3, \mathrm{~b} 1, \mathrm{~b} 3)$, and $\mathrm{U}(\mathrm{b} 1,0,0), \mathrm{U}(\mathrm{b} 2, \mathrm{a} 1, \mathrm{a} 2), \mathrm{U}(\mathrm{b} 3, \mathrm{a} 3,0)$;

(5) step: $\mathrm{M}(\mathrm{a} 1, \mathrm{~b} 2, \mathrm{~b} 1)$, and $\mathrm{U}(\mathrm{b} 1, \mathrm{a} 1,0), \mathrm{U}(\mathrm{b} 2, \mathrm{a} 2,0), \mathrm{U}(\mathrm{b} 3, \mathrm{a} 3,0)$;

(6) step: $\mathrm{M}(\mathrm{a} 2, \mathrm{~b} 2, \mathrm{~b} 3)$, and $\mathrm{U}(\mathrm{b} 1, \mathrm{a} 1,0), \mathrm{U}(\mathrm{b} 2,0,0), \mathrm{U}(\mathrm{b} 3, \mathrm{a} 2$, a3);

(7) step: $\mathrm{M}(\mathrm{a} 1, \mathrm{~b} 1, \mathrm{~b} 3)$, and $\mathrm{U}(\mathrm{b} 1,0,0), \mathrm{U}(\mathrm{b} 2,0,0), \mathrm{U}(\mathrm{b} 3, \mathrm{a} 1, \mathrm{a} 2)$;

(8) the goal: $\mathrm{U}(\mathrm{b} 1,0,0), \mathrm{U}(\mathrm{b} 2,0,0), \mathrm{U}(\mathrm{b} 3, \mathrm{a} 1, \mathrm{a} 2), \mathrm{U}(\mathrm{b} 3, \mathrm{a} 2, \mathrm{a} 3)$.

Of course, this is not a unique representation, and there exists a lot of other ways to represent it (Feng Ding, 2006; Zhang Xiao-jiang, Liu Chang, 2005).

\subsubsection{The limitation of knowledge representation}

Knowledge representation is the arithmetic model of intelligence simulation. It does not explore the nature of intelligence or define inner process of human intelligence, especially for traditional symbolism (Wei Hui, Pan Yun-he, 2000). Though we can use predicate logic to describe the Tower of Hanio clearly, it is not the real way in which brain's solving process works. We can prove this by analyzing the phases of programming.

The program is a typical knowledge task. The program can be divided into two phases. In the first phase, the programmer should solve his own cognitive problem using natural language to understand the aim of the program. The second phase is to solve the cognitive problem of the computer (Li Yong-jian, Wang Lei et al, 2006). By coding knowledge into a form that the computer can understand makes the computer fulfill the task needed for intelligence. The choice of how to represent knowledge reflects the developer's cognition of simulated intelligence behavior. But not every representation is the real reflection. Both the symbolism and connectionism are only the approximate representation of the mental process. The mind operation focuses on the mechanism of intelligence, pursuing the objective reality (Wei Hui, Pan Yun-he, 2000). We can get to the new cognition of the intelligence by exploring the biological and psychological process of intelligence itself. The new way to study the subject indicates the advanced in the methodology of AI (Wei Hui, Pan Yun-he, 2000).

The knowledge presentation in AI can solve the cognitive problem of the computer, and provides the indirect way to describe the process of knowledge work. But it can't solve the process management problem in knowledge task, such as optimization and measurement. So, we should find the more direct way to describe the knowledge task process.

Here we use the cognitive process theory and mind operation to describe the knowledge work process.

\subsection{The MO representation of the knowledge task process}

\subsubsection{The knowledge task process is "a series of activities" (a series of MO's)}

Before classic scientific management, the process of manual movements was a "black box" similar to knowledge tasking. Since Taylor and Gilbreth brought forward the concepts of "basic movement" and "therbligs", manual processes began the "white box" which can be described and measured. This became the foundation of scientific management. If we describe the process of knowledge task by activities, the inner process of it can turn the "black box" into a "white box". In cognitive science, the activity is the MO.

$\mathrm{Wu}$ wenjun, the academician who achieved the highest scientific technology honor of china, considers the most probable subject to be mechanized should be mathematics. That is the typical mental labor. In fact, math is stricter, more concise and valuable than other subjects. So math is easier to be mechanized than other mental labor. In the view of $\mathrm{Wu}$, take the problem (1) as an example. The task is to identify as many mental functions as possible; the requirement is that the addition of two digits is equal to the product of the two digits.

$$
4 \frac{1}{2}+1 \frac{2}{7}=4 \frac{1}{2} \times 1 \frac{2}{7}
$$

According to 3 persons thinking aloud, we analyze the process of MO as follows:

Step 1: information perception. To identify what kind of problem this is: addition and multiplication.

Step 2: information extracting. Activate relative knowledge and experience for the addition and multiplication information stored in the brain.

Step 3: problem identification. Carify the problem and requirement: make the addition of the two digits equal to the product of the two digits.

Step 4: Transformation. According to previous knowledge about fractions, we transform the example (1) into (2) below

$$
4 \frac{1}{2}+1 \frac{2}{7}=4 \frac{1}{2} \times 1 \frac{2}{7} \Rightarrow \frac{9}{2}+\frac{9}{7}=\frac{9}{2} \times \frac{9}{7}
$$


Step 5: character comparing. Compare the two numerators and denominators, and try to find the similarity and difference.

Step 6: character extracting. The two numerators are the same, and the two denominators are different.

Step 7: reasoning. The addition of two denominators is equal to the numerators.

Step 8: Character conformity. The common numerator (9) of the two improper fractions is equal to the addition of the two denominators $(2+7)$.

Step 9: supposing. If the common numerator of the two improper fractions is equal to the addition of the two denominators, the product of the two fractions will be equal to their addition.

$$
\text { If } a+b=k, \text { then } \frac{k}{a}+\frac{k}{b}=\frac{k}{a} \times \frac{k}{b}
$$

Step 10: validating. According to the supposing, find some example to validate.

$$
2 \frac{1}{4}+1 \frac{4}{5}=2 \frac{1}{4} \times 1 \frac{4}{5}
$$

Step 11: symbolic describing. To describe logical thinking in nature or artificial language.

Step 12: standardization. Standardizing the result of step 10, and program as follows.

Void $\mathrm{f}$ (int $\mathrm{x}$, int $\mathrm{y}$ )// define the function containing the two parameters to fulfill the requirement of task (1) \{

if $(x>0 \& \& y>0) / /$ the two digits are positive number.

\{

int $\mathrm{z}, 1, \mathrm{~m}, \mathrm{n}, \mathrm{p} ; / /$ define the assistant parameters;

$\mathrm{z}=\mathrm{x}+\mathrm{y} ; / /$ ascertain the common numerator;

$\mathrm{l}=\mathrm{z} / \mathrm{x} ; \mathrm{m}=\mathrm{z} \% \mathrm{x} ; / /$ transform the first proper fraction into mixed number;

$\mathrm{n}=\mathrm{z} / \mathrm{y} ; \mathrm{p}=\mathrm{z} \% \mathrm{y} ; / /$ transform the second proper fraction into mixed number;

if(m\&\&p)//when the two are all in fractional forms

\{

$\operatorname{printf}(“ \% \mathrm{~d}(\% \mathrm{~d} / \% \mathrm{~d})+\% \mathrm{~d}(\% \mathrm{~d} / \% \mathrm{~d})=\% \mathrm{~d}(\% \mathrm{~d} / \% \mathrm{~d}) * \% \mathrm{~d}(\% \mathrm{~d} / \% \mathrm{~d}) \backslash \mathrm{n} ", 1, \mathrm{~m}, \mathrm{x}, \mathrm{n}, \mathrm{p}, \mathrm{y}, 1, \mathrm{~m}, \mathrm{x}, \mathrm{n}, \mathrm{p}, \mathrm{y}) ; / /$ output the result \}

else if(!m\&\&!p)//when both of then are positive integers

\{

$\operatorname{printf(“\% } \% \mathrm{~d}+\% \mathrm{~d}=\% \mathrm{~d} * \% \mathrm{~d} \backslash \mathrm{n} ", 1, \mathrm{n}, 1, \mathrm{n})$;

\}

else if(!m)//when the first is positive integer

\{

$\operatorname{printf(}(\% \mathrm{~d}+\% \mathrm{~d}(\% \mathrm{~d} / \% \mathrm{~d})=\% \mathrm{~d} * \% \mathrm{~d}(\% \mathrm{~d} / \% \mathrm{~d}) \backslash \mathrm{n} ", \mathrm{l}, \mathrm{n}, \mathrm{p}, \mathrm{y}, \mathrm{l,n}, \mathrm{p}, \mathrm{y}) ;$

\}

else// when the second is positive integer

printf(“\%d(\%d/\%d)+\%d=\%d(\%d/\%d)*\%d $\%$ n", $1, m, x, n, 1, m, x, n$,

\}

else

printf ("They can't be formed a proper formulation");// the other situations.

\}

3.2.2 The necessary declaration

What should be pointed out is that even for the same knowledge task, each person has a different mind operation chain. 
What MOs people will use are not just limited to the ones mentioned above, and the sequence of the MO in the chain is not unique. Different types of knowledge works have the different MOs, and the same MO in different types of knowledge task is not in the same position of the chain, just like the therbligs in manual labor (Wang Jin-hua, 1997).

Is MO ubiquity in knowledge task? What are the common MOs in knowledge task? We will try to use the research to test the ubiquity of MO and its ability to use the MO to explain the process of knowledge task.

\section{The confirmatory research on MO}

\subsection{Research purpose}

To explore whether observed data will conform to the mind operation concepts set model (see fig.2) by LISREL (Linear Structural Relations). Confirmation requires the following: First, whether there exist MO elements in knowledge task process. Second, whether there is a proper structure of the elements.

\subsection{Research method-The introduction of Structure Equation Model (SEM)}

SEM is a universal and primary linear statistic modeling technology. It is the combination of statistic analysis methods in the fields of econometrics, sociometrics and psychometrics. SEM also uses simultaneous equations to ask for answers, but it doesn't require strict assumptions or limitations, and simultaneously, it allows both independent and dependent variables to exist. SEM is primary a confirmatory technology. It is used to ensure that a certain model is reasonable but not to seek and discover a suitable model for researchers. In our research, we use SEM to confirm whether our hypotheses are reasonable.

There are five primary steps when using SEM (Bollen, Kenneth, J. Scott Long, 1993):

(1) Model specification: according to theory or former research, researchers need to specify the assumptive initial theoretic model before model estimation.

(2) Model identification: this step determines whether the research model has the only answer of parameter estimation. In some situations, as the model is specified mistakenly, the parameters can't be identified and it can't get the only estimation values. As a result, the model has no answer.

(3) Model estimation: the model parameters can be estimated with several different methods. The most popular model estimation methods are maximum likelihood and generalized least squares.

(4) Model evaluation: After the acquirement of parameter estimation values, it needs to appraise whether the model and data are fit, and compare with alternative model's fitness indices.

(5) Model modification: If model can not fit well to the data, the model must be modified and specified again. In such situations, the researcher needs to determine how to delete, add and modify the parameters of model. Through parameters re-specification, it can improve model's degree of fitness. Researcher can determine the model's re-specification according to the model modification indices provided by LISREL soft and the results of test of initial model paths. Once model is specified again, it needs to repeat the five steps above. A model that fits well usually repeat these steps many times.

The five steps above are the groundwork when researching a theoretic model by SEM. Especially, the confirmatory factor analysis (CFA) is also a type of SEM method.

\subsection{The hypothesis for the concept model of the MO}

In light of the analysis from the point of view of both the AI and cognitive psychology, we can regard the process of knowledge task as the process of information symbol transformation (Simon H.A, 1986). In the view of modern cognitive ergonomics, knowledge tasking is the reasonable sequence of MO (Wang Jin-hua, 1997).

The Gilbreth's initiated the method that screened and analyzed the movements of task with cine-cameras and calculagraphs. It broke down human being's task movements into three types with seventeen basic motions in all (named "Therbligs"). They were: stretching, grasping, moving, assembling, applying, disassembling, unclenching, checking, searching, choosing, planning, aiming, pre-aiming, holding, resting, staying and delaying (Frank B. Gilbreth, 1912).

WF (Work Factor) divided human operational movements into eight types under the consideration of four variables - the body parts, move distance, manual control level and resistance or weight. They were carrying, snatching, pre-aiming, assembling, using, disassembling, brain processing and unclenching.

MTM (Methods Time Measurement) divided human operational movements into stretching, carrying, rotating, pressing, running, grapping, orientating, unclenching, disassembling, eye-moving and body-movement and so on. By analyzing the variables of movement level, movement distance, movement form, resistance or weight, movement type or character, and together with simultaneous motion, combined motion, compound motion scene, it included three forms of MTM-1, MTM-2 and MTM-3, based on the movement's degree of accuracy. 
In 1959, Wofac Corporation took the lead to put forward the method of Variable Factor Program (VFP), which studies the brainwork. In 1967, it brought forward Wofac Mento-Fator System (WMFS) based on Predetermined Motion-Time Study (PMTS). WMFS had determined the task element system which included fourteen elements: sight movement, sight watching, looking, nerve transmitting, distinguishing, estimating, identifying, deciding, remembering, recalling, calculating, confirming, transforming and attention transferring.

Using the perspective of the Scientific Management's process the analysis of knowledge task process can be thought of being comprised of "a series of" mind operations which are similar to the "basic motions" or "Therbligs". From the perspective of cognitive science, knowledge task is an operational (transforming) process of the mind on information symbols. The mind's operation is an algorithm of knowledge tasking (transformative rule). Based on these definitions, the mind's operation can be viewed as "operational modes of information processing", while knowledge task process is the set of these information-processing modes (mind operations). We can then present simple manipulative definitions of mind operation concepts on the "therblig" level as follows:

(1) Metacognition. Simply speaking, metacognition is cognition and adjustment on cognitive activity processes. It plays a role in constituting plans, choosing strategies, supervising execute, appraising results and adjusting feedback. It is the highest-level control or fountainhead of the following MOs.

(2) Distinguishing. The ability to discern and confirm expressive forms of information, every kind of perception, discrimination and recognition. Its meaning is similar to the "cognize" defined by Guilford.

(3) Memory. Maintains existing knowledge, searches and memorizes new information.

(4) Transforming. Changes the forms of information. For example, transform letter information into table information.

(5) Imagining. Changes and transforms images into new one. For example, we read the ancient poetry sentence "Flocks and herds appear as grass bends to wind"; it would appear a beautiful picture in our brain. This is the result of imagination. When we imagine, we all depend on the images already stored in our brain.

(6) Character extracting. Breaking down the whole object (cognitive object) into parts and extract the characters of cognitive objects. It has three levels: (1)action thinking level, such as tearing down the clock's parts one by one within brain; (2)imagery thinking level, such as breaking down the image of a tree into roots, branches and leaves; (3)abstract thinking level, such as disassembling the chemistry equation.

(7) Character integrating. Combining the parts of the cognitive objects, i.e., characters, attributes, within the brain. "Character integrating" and "character extracting" are both dialectic mind operations. They also have three levels: (1) motion thinking level, such as assembling the clock's parts one by one within the brain; (2)imagery thinking level, such as synthesizing the image of roots, branches and leaves into a tree within the brain; (3)abstract thinking level, such as combining and establishing simultaneous equations.

(8) Reasoning. Concluding from known or assumptive facts, or inferring a new judgment from one or more known judgments.

(9) Concretization. Applying general conclusions to idiographic things; it is a type of deduction.

It is necessary to explain two points about the MO enumerated above. First, MO's can be subdivided by degree and whether or not they are dependant on practical demands and conditions. Second, it can define and add new MO's. This is very much like the differences and specialties among MTM, WF and MOD in IE (Industry Engineering).

According to the theories of cognition science and the definitions given above, together with Structural Equation Model and confirmatory factor analysis (Barbara M. Byrne, 1998), the structure of the MO concepts set could be considered as a full model, showed in Figure 2.

Insert Figure 2 here

Metacognition is the highest level processing operation. It plays a role on planning, supervising and adjusting to the other mind operations in the whole knowledge task process. So, metacognition is an exogenous latent variable $(\xi)$, the other mind operations are all endogenous latent variables $(\eta)$ of metacognition (Sun Shang-gong, 2002). $X_{i}$ and $Y_{i}$ are vectors comprised of $\xi$ and $\eta$ 's observational indices respectively.

\subsection{The design of the questionnaire}

According to the frame of scales' development process, Spector subdivided Likert's three stages into five steps (Rhonda L. Hensley, 1999): (1) define the construct; (2) design the scale; (3) pilot test the scale, confirming the exact connotation of all items; (4) administer the scale and perform item analysis to determine whether the items form scales. Examine and emend the items' intelligibility; (5) validate and normalize the measures.

The structure of the questionnaire is consistent with the theoretical hypothesis framework. It presents the operational definition of every observed variable. The nine basic mind operations compose nine dimensions of the questionnaire 
and each dimension includes three to six items. There are three sources of the questionnaire items' content. First, the theoretical research of scientific management, cognitive science and intelligence; second, the connotation of each mind operation in knowledge task process; third, the results of previous surveys and small sample pretesting. In order to ensure that the population or subjects understood the meaning and content of each item, we examined the intelligibility of the mind operation with each item. They all reached the level of "comparative understanding easily" or better. For example, for the factor of metacognition we designed 5 items of observed variable. Some items were as follows: "Examine the correctness of thought when analyzing and solving problem $\left(\mathrm{x}_{1}\right)$ ", "Adjust and control the thinking process consciously when analyzing and solving problem $\left(\mathrm{x}_{2}\right)$ ", "Appraise one's own work processes, methods and results $\left(\mathrm{x}_{3}\right)$ ". Then we tested the comprehensibility of the items. All items tested above the "easy to understand" level. This guaranteed the exact understanding of each item.

According to the purpose and hypothesis of the research, we apply the five-point Likert scales. The dimension of questionnaire was frequency of use: often, many, unclear, less, once in a while.

\subsection{The collection and summary of the data}

We used the questionnaire to collect the data which verified that knowledge task is the mechanism of brainwork, and knowledge task processing is the information processing of human the brain. For this study, we chose workers that perform mental (brain) functions and especially software engineers as subjects. This was done considering that programming tasks is a kind of typical knowledge process (Wang Lei, Li Yong-jian, 2006).

The sources of subjects in this study were students in the following programs: Master of Software Engineering (MSE) in University of Electronic Science and Technology of China (UESTC), Master of Business Administration (MBA) in UESTC. 235 questionnaires were distributed and 191 completed questionnaires were returned. In these valid questionnaires, there were 118 MSE and 73 MBA; 143 males', 36 females' and 12 people didn't fill "sex".

The data used SPSS11.5 for statistical processing and LISREL8.53 for confirmatory factor analysis (CFA). We eliminated four items whose least component load was below 0.36. 41 items were processed using LISREL8.53 analysis and the data samples composed by the 191 subjects was processed within the LISREL8.53 - according to the structural equation method.

\section{Study results}

\subsection{Results and Discussion}

We inputted the covariance matrix of target variables (measurement variables), the affiliation of targets and latent variables (namely, the structure). Then the software package LISREL (Joreskog K. G, Sorbom D, 1993) was able to estimate the parameters, such as the relationships of targets and latent variables, latent variables and latent variables, the parts which the model cannot explain and the measurement error of targets, and so on. The values of these parameters could reflect the strength of each relationship. Besides, the software package could compute whether the model put forward by the researchers fit to the sample data (whether the model could express the data) (Hau K. T, Cheng Z. J, Chung C. M, 1996; Kong C. K, Hau K. T, 1997; Marsh H. W, Hau K. T, 1996).

The resultant structure included two highly correlated aspects; the elements of structure and relationships among these elements. According to the study's theoretical analysis and confirmatory analysis based on structural equation method, using the LISREL8.53 program and maximum likelihood estimation, the research gained the SEM fit indices results of observed data, shown in Table 1.

Insert Table 1 here

The program chose fit indices as follows: Chi-square $\left(\chi^{2}\right)$, NNFI and CFI (critical value is 0.9 ), RMSEA (critical value is 0.08) (Wen Zhong-lin, Hau Kit-Tai, Herbert W. Mars, 2004). $\chi^{2}$ goodness-of-fit test is the most often used measure standard about a models degree of total fit. According to the critical values of each index, the data in Table 1 showed that the structural equation model described in Figure 1 fit the observed data; the fit indices were comparatively perfect. In this paper, RMSEA equaled 0.06 and less than 0.08 (RMSEA $=0.06<0.08$ ). The absolute index RMSEA weighed the fit degree between the theoretical model and sample data (Wen Zhong-lin, Hau Kit-Tai, Herbert W. Mars, 2004). This indicated that observed data's ability to interpret knowledge task process with a 9-factors model showed in figure 1 . The knowledge task process of existing nine factors and the structural relation which metacognition controlled the other mind operations was also verified. Knowledge task process can be interpreted by mind operational concept structure showed in Figure 1. The nine factors were comprised of metacogniton and other eight factors controlled by metacognition. The structure of mind operation concepts set is shown in figure 3 below.

Insert Figure 3 here

In practice, mind operations are always highly related to operational objects, and always operates on the object in certain conditions and environment. So, the expressive forms of mind operation are various (like the intelligence's complex forms). They are numerous, complex and observed variables of mind operations (this paper has confirm 41 
entries in confirmatory analysis). Through confirmatory analysis, this paper sums up in variable and un-reductive components from the diverse process and phenomena of knowledge task, and uses them to verify the structural form and related hypothesis (Figure 2). The final results of the model showed in Figure 3. Exogenous latent factor metacognition was the "upriver" variable of nine endogenous latent variables; it indicated that metacognition played a role on planning, supervising, adjusting and controlling. This agreed with the perspective of cognitive science and intelligence theory.

\subsection{Conclusion and implications}

This study reached the following conclusions and implications

1) $\mathrm{AI}$ and knowledge engineering provide an indirect way to describe the process of knowledge tasks. But, it cannot solve the problem of how to manage the process, for example, the optimization and measurement of the tasks. We must explore a direct way to describe the process of knowledge work. This is not only the requirement of the knowledge task management, but also indicates advancement in AI (Wei Hui, Pan Yun-he, 2000).

2) The confirmatory research indicates: the MO set that can represent the process of knowledge task is composed by 9 MOs and the 8 elements that are controlled by metacognition very much as the "therblings" in MTM, WF and MOD. Which elements are parts of the MO varies according to practice (Wang Jinhua, 1997).

3) The optimization of knowledge task is achieved through the reasonable design of the "sequence of MOs" (Wang Jinhua, 1997). This is similar to the optimization of manipulation tasks required to design the sequence of "therblings". To optimize the MOs of knowledge tasks requires further study of more and different knowledge tasks in order to explore and conclude principles and methods.

4) According to the principle of PTS in IE we can relate the process of knowledge work to MOs and provide the object to be measured. We can consider measuring the "MOD" of the MO or the "intelligence quotient" of MO.

In summary, in depth exploration and standardization of the MO representation of knowledge task is a potential way to realize the scientific management of knowledge tasks. It also indicates an advancement in the methods of AI (Wei Hui, Pan Yun-he, 2000). All discussions about process management knowledge tasks have fundamental theory significance both in knowledge management and AI (Peter F. Drucker, 1999; Wang Jin-hua, 1997).

\section{References}

Barbara M. Byrne. (1998). Structural equation modeling with Lisrel, Prelis, and Smplis: base concepts, applications, and programming. LAWERNCE ERLBAUM ASSOCIATES, PUBLISNERS MAHWAH, NEW JERSEY.

Bollen, Kenneth, \& J. Scott Long. (1993). Testing Structural Equation Models. New Bury Park: Sage.

Chen Lin. (1993). The progress in the research of some fundamental problems of cognitive science. Journal of Chinese Academy of Science (in Chinese), (2): 112-18.

E. Kevin Kelloway, \& Julian, Barling. (2000). Knowledge work as organizational behavior. International Journal of Management Reviews, Volume 2, Issue 3:287-304.

Feng, Ding. (2006). Expert System of Artificial Neural Network. Beijing: Science Press (in Chinese), 136-186.

Frank B. Gilbreth. (1912). A paper presented at 1912 American Society of Mechanical Engineers (ASME) Meetings.

Frederick W. Taylor (1911). The Principles of Scientific Management. New York: Harper-Row Publishing House.

Fu, Xiaolan \& Liu, Chao. (2003). Approaches and Methods in Cognitive Psychology to Explore the Mind. Journal of Dialectics of Nature (in Chinese), Vo1.25, No. 5, 96-101.

Hau K. T, Cheng Z. J, \& Chung C. M. (1996). Issues in fitting and a comparison of common indexes in structural equation modeling. Hong Kong Education Research Journal, 11: 73-81.

Hau Kit-Tai, Wen, Zhong-lin \& Cheng, Zijuan. (2004). Structural Equation Modeling and Its Application (in Chinese). Beijing: Educational Science Publishing House.

Joreskog K. G, Sorbom D. (1993). LISREL 8 User's Reference Guide. Chicago, IL: Science Software International.

Kong C. K, \& Hau K. T. (1997). Issues and misconceptions in using structural equation modeling. Education Journal, 25:45-60.

Li Yong-jian, \& Ma, Shun-dao (2004). Analysis of "Philippe" in Software Engineering. Computer Engineering and Applications (in Chinese), Vol.40, 57-59.

Li, Yong-jian, Wang Lei, Wang Bo \& Tiang Qing. (2006). Research on the Representation of the Programming Task Process, The 36th International Conference On Computers and Industrial Engineering (ICCIE), Taipei: National Tsing Hua University Press, 13. 
Liu, Zhongpei \& Sun, Jing. (2001). From Existence to Evolution: the Change of Paradigm of Artificial Intelligence Research and Its Philosophic Idea. Journal of Systemic Dialectics (in Chinese), Vol.9, No. 2:71-76.

Marsh H. W, \& Hau K. T. (1996). Assessing goodness of fit: Is parsimony always undesirable? The Journal of Experimental Education, 64:364-390.

Minsky M. (1968). Semantic Information Processing. Mass: MIT Press, 5-10.

Peter F. Drucker. (1999). Knowledge-worker Productivity: The Biggest Challenge. California Management Review, Winter, 79-94

Peter F. Drucker. (1999). Management Challenges for the 21st Century. New York: Harper Business.

Rhonda L. Hensley. (1999). A review of operations management studies using scale development techniques. Journal of Operations Management, 17:343-358.

Russell Curtis, \& David Leon. (2002). Supporting knowledge work with physical design. Knowledge Management Review, Volume 5, Issue 5:26-32.

Simon, H.A. (1983). Search and reasoning in problem solving. Artificial Intelligence, 21, 7-29.

Sternberg R. J. (1985). Beyond IQ: A Triarchic Theory of Human Intelligence. New York: Cambridge University Press.

Sun, Shanggong. (2002). A brief introduction of latent variables analysis-1. Applications of Statistics and Management (in Chinese), Vol. 21, (1): 52-58.

Tan, Haoqiang. (1999). The C Programming Language(2nd Edition). Tsinghua University Press (in Chinese), 161-163.

Thomas H. Davenport, Robert J. Thomas, Susan Cantrell. (2002). The Mysterious Art and Science of Knowledge-Worker Performance. MIT Sloan Management Review, Fall, 23-31.

Thomas H. Davenport, Sirkka L. Jarvenpaa, \& Michael C. Beers. (1996). Improving Knowledge Work Processes. Sloan Management Review, 26(10):53-66.

Tomas Hellstroèma, Ulf Malmquistb, Jon Mikaelssonc (2001). Decentralizing knowledge: managing knowledge work in a software engineering firm. Journal of High Technology Management Research, Vol.12 (1): 25-38.

Wang, Jinhua. (1997). Military Ergonomics. Beijing: Defense University Press (in Chinese).

Wang, Lei, \& Li Yongjian. (2006). Mind Operation Description of Software Programming Task Process. Computer Engineering and Application (in Chinese), Vol.42 (4):33-38.

Wang, Qianling. (2004). The Change of Research Guidelines of AI. Journal of Social Science Research(in Chinese), (6):54-60

Wei, Hui, \& Pan, Yunhe. (2000). The Progress in Epistemology of AI: from Knowledge Representation to Representation. Journal of Computer Research and Development (in Chinese), Vol. 37, No. 7, 819-826.

Wen, Zhonglin, Hau Kit Tai, \& Herbert W. Marsh. (2004). Structural Equation Model Testing: Cutoff Criteria for Goodness of Fit Indices and Chi-Square Test (in Chinese). Acta Psychologica Sinica, 36(2):186-194.

Zhang, Xiaojiang, \& Liu, Chang. (2005). The Cognitive Process in Tower of Hanoi Solving and Its Feature. Studies of Psychology and Behavior (in Chinese), 3(1):44-49.

Table 1. Fit indices values between theoretical hypothesis and observed data

$$
\chi^{2} / d f \quad \text { NNFI IFI CFI RMSEA }
$$

$\begin{array}{lllll}1.669 & 0.916 & 0.924 & 0.924 & 0.0593\end{array}$

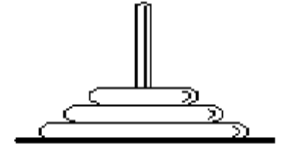

b1
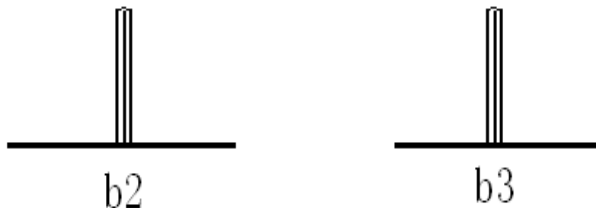

Figure 1. Sketch map of "tower of Hanoi" 


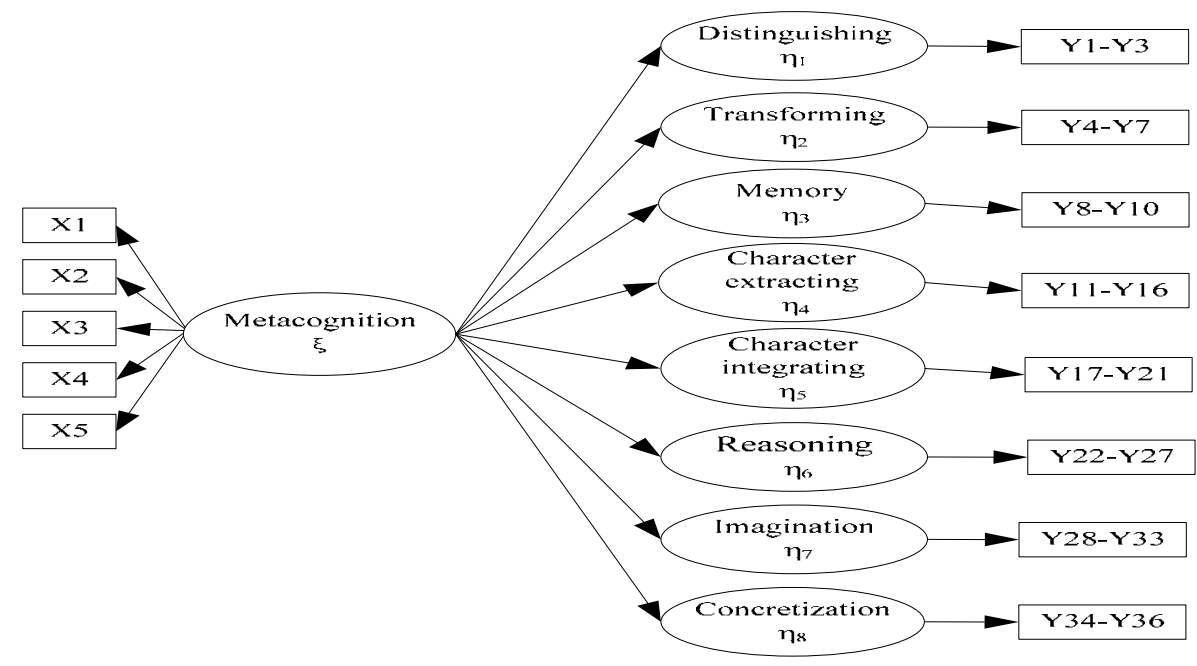

Figure 2. Model of Mind Operation concepts for describing the knowledge task process

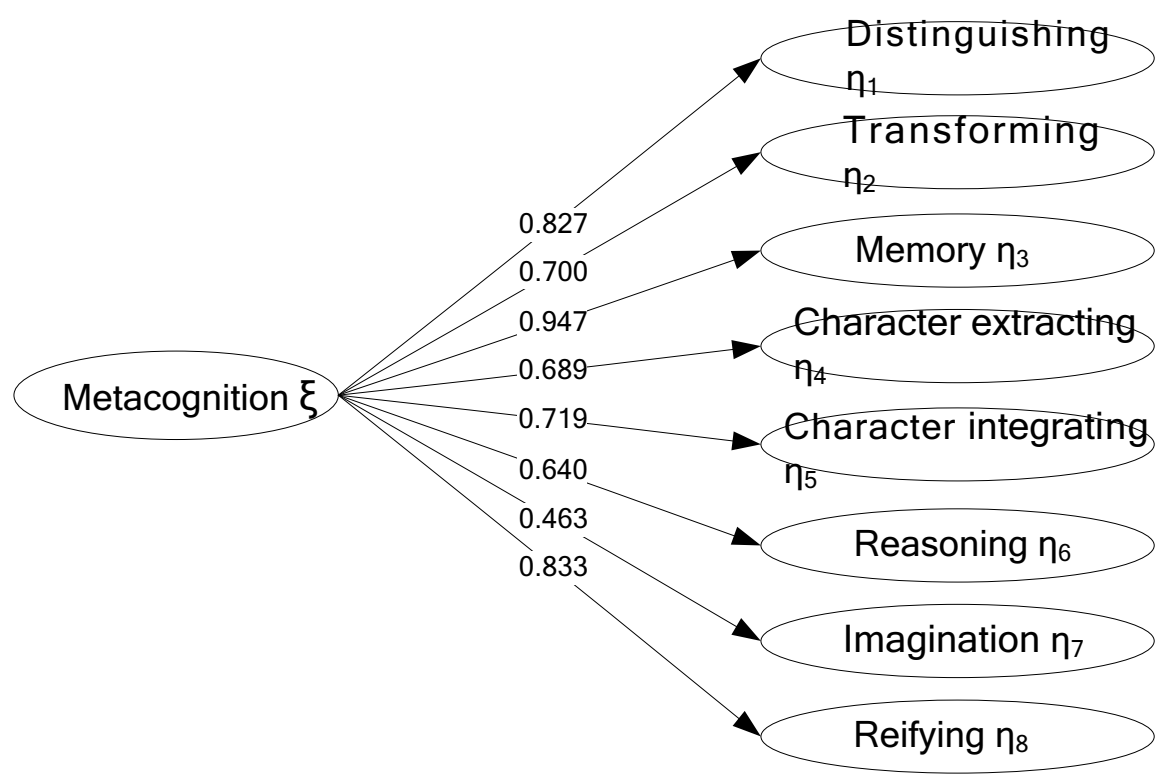

Figure 3. Structure of mind operation concepts set 